\title{
INTERACTIVE EFFECTS OF TRAIT AND STATE ANXIETY ON VISUAL SPATIAL WORKING MEMORY CAPACITY
}

\author{
Jun MORIYA
}

Kansai University, Japan

\begin{abstract}
Anxiety is associated with low visual spatial working memory capacity (VSWMC) in the presence of task-irrelevant distractors. Because anxious individuals have difficulty suppressing processing of distractors, VSWMC is depleted. However, results of association between anxiety and VSWMC in the absence of distractors are inconsistent among previous studies. In the present study, distinct roles of trait and state anxiety were focused. The present study investigated the interactive effects of trait and state anxiety on VSWMC without distractors using a change detection task. In the task, a memory array of oriented bars to be encoded was presented followed by a test array, and participants were required to answer whether the test array was identical to or different from the memory array. Orientation of one bar changed largely (i.e., low-precision condition) in Experiment 1 and slightly (high-precision condition) in Experiment 2. In Experiment 1, the interactive effects of trait and state anxiety on VSWMC were observed. VSWMC was positively associated with state anxiety when trait anxiety was low, whereas VSWMC was negatively associated with state anxiety when trait anxiety was high. These interactive effects were not observed under high-precision condition in Experiment 2, in which VSWMC was negatively correlated with state anxiety irrespective of trait anxiety. These results indicated that under low-precision condition, high state anxiety widely allocate working-memory resources and VSWMC becomes high. However, because high trait anxious individuals have difficulty controlling allocation of working-memory resources, high trait anxiety decreases VSWMC under high state anxiety. Under high-precision condition, it is necessary to allocate working-memory resources narrowly to detect slight change. Because of wide allocation of working-memory resources in high state anxious individuals, state anxiety is negatively correlated with VSWMC under high-precision condition.
\end{abstract}

Key words: state anxiety, trait anxiety, visual spatial working memory capacity, moderation

Anxiety restricts working memory capacity (WMC; Moran, 2016). Anxious thought depletes WMC and residual WMC in anxious individuals is low (Hayes et al., 2008; Leigh \& Hirsch, 2011). Because of low WMC, these anxious individuals have difficulty maintaining task-relevant, neutral information in working memory (Amir \& Bomyea, 2011). Moreover, considering that lower WMC predicts impairment of other cognitive functions, such as emotional regulation (Yoon et al., 2018), it may be a risk factor for anxiety. Moran's (2016) meta-analysis revealed that low WMC was associated with anxiety in almost all working-memory tasks - including the operation span task, the

Correspondence concerning this article should be addressed to Jun Moriya, Faculty of Sociology, Kansai University, Osaka, Japan (e-mail: jmoriya@kansai-u.ac.jp). 
simple span task, and the dynamic span task-but not concerning visual spatial WMC (VSWMC).

VSWMC is defined as the active maintenance of visual features, such as oriented bars, color patches, symbols, or objects, to help recall features and locations later for ongoing tasks (Luck \& Vogel, 1997, 2013). The change-detection task is a widely used way to calculate VSWMC. In this paradigm, a memory array of visual stimuli is presented followed by a test array after a blank delay, and participants indicate whether the test array is identical to or different from the memory array (Luck \& Vogel, 1997). In many tasks using the paradigm, task-irrelevant distractors are also presented. Because it is not necessary to encode the task-irrelevant distractors, participants are required to suppress their processing. Many previous studies have consistently shown that anxious individuals have difficulty suppressing distractors (Berggren et al., 2017; Moriya \& Sugiura, 2012; Qi, Ding, \& Li, 2014; Stout \& Rokke, 2010). Moreover, anxious individuals display low VSWMC because VSWMC comprises task-irrelevant items. However, when task-irrelevant distractors are not presented, the association between anxiety and VSWMC is inconsistent. Some studies indicated negative associations (Berggren et al., 2017; Qi, Chen, et al., 2014), while others reported near-zero associations (Qi, Ding, \& Li, 2014; Stout \& Rokke, 2010) or even positive associations (Moriya, 2018). Thus far, no studies have explained these inconsistent results.

One possibility is that trait and state anxiety distinctly influence VSWMC. Previous cognitive models concerning anxiety proposed distinct roles of trait and state anxiety in cognitive processing, especially attentional processing (Bishop, 2007; Eysenck \& Derakshan, 2011; Eysenck et al., 2007; Williams et al., 1988). According to attentional control theory (Eysenck \& Derakshan, 2011; Eysenck et al., 2007), bottom-up visual attention to salient stimuli is enhanced in individuals with high state anxiety, whereas top-down control of visual attention is impoverished among individuals with high trait anxiety. Bishop (2007) also proposed a similar neurobiological model, according to which elevated state anxiety increases amygdala activation for automatic threat detection, or bottom-up affective processing, whereas trait anxiety impairs top-down attentional control in the prefrontal cortex (PFC). The results of several previous behavioral and neuroimaging studies supported these two models. Pacheco-Unguetti et al. (2010) compared the attentional functions of trait and state anxiety using an attention network test. They indicated that high trait-anxious individuals showed impaired executive attention, which is associated with top-down control. During high state anxiety under anxious-mood induction, on the other hand, individuals showed enhanced alerting and involuntary orienting, which are associated with bottom-up processing. In neuroimaging studies, when conducting cognitive tasks (e.g., visual search task, Stroop task, etc.), high trait anxiety was associated with reduced activation of the dorsolateral PFC (DLPFC), which is related to top-down control. However, high state anxiety was associated with elevated activation of the amygdala and superior temporal sulcus, indicating bottom-up processing (Bishop, 2009; Bishop et al., 2007; Crocker et al., 2012). These results supported that trait anxiety is associated with impaired top-down control and state anxiety is associated with enhanced bottom-up control. 
In addition, the combination of trait and state anxiety is also important for cognitive functions in anxiety. Several studies have suggested that both high trait and high state anxiety reduce top-down cognitive control (Egloff \& Hock, 2001; Hur et al., 2015; Rutherford et al., 2004). Poor performance due to Stroop interference was observed in the presence of a negative state and trait affect including anxiety. Crocker et al. (2012) also revealed that, during an emotional Stroop task, increased trait negative affect was associated with reduced activation of the DLPFC during high state anxiety. On the other hand, low-trait-anxious individuals might show increased top-down control, such as executive function, in high state-anxiety situations (Ursache \& Raver, 2014). In threatening conditions, individuals with low trait anxiety display high functional connectivity between the ventromedial PFC and the amygdala (Bijsterbosch et al., 2015). That is, low trait-anxious individuals efficiently utilize top-down control under high stateanxiety conditions to control the situation. Taken together, the impoverished top-down processing is observed only in high trait-anxious individuals during high state anxiety. In low trait-anxious individuals, on the other hand, high state anxiety enhances bottom-up processing and top-down control.

The above explanation also fits for VSWMC. Previous studies have suggested that top-down control influences VSWMC (Cusack et al., 2009; Linke et al., 2011). When many stimuli (e.g., eight items) are presented in the change-detection task, workingmemory performance is depressed compared with when only a few stimuli are presented because VSWMC is limited to three or four items (Cowan, 2001; Luck \& Vogel, 1997). In such situations, it is efficient to utilize top-down control to retain a restricted number of stimuli (Cusack et al., 2009; Linke et al., 2011), thus increasing VSWMC. That is, VSWMC will decrease in individuals with impaired top-down control because poor attentional selection cannot make people selectively encode the stimuli. Considering that impoverished top-down processing is observed in high trait-anxious individuals during high state anxiety, VSWMC would be expected to decrease in high trait- and stateanxious individuals. On the other hand, because high state anxiety enhances top-down control in low trait-anxious individuals, they would be expected to show high VSWMC during high state anxiety. The results of previous studies, in which trait anxiety was positively correlated with VSWMC without any anxiety induction, are partially consistent with this idea (Moriya, 2018; Moriya \& Sugiura, 2012). However, those studies did not measure state anxiety.

The current aim was thus to clarify the interactive effects of trait and state anxiety on VSWMC using a change-detection task. As described above, impaired top-down control is observed in high trait- and state-anxious individuals. Therefore, I hypothesized that low VSWMC would be observed in high trait- and state-anxious individuals. On the other hand, high state anxiety enhances top-down control in low trait-anxious individuals. Thus, I also hypothesized that high VSWMC would be observed in individuals with low trait but high state anxiety. In the present study, trait and state anxiety were measured using scales without mood induction after the change-detection task. Because questionnaires which are administered before cognitive tasks have an effect on performance of the following tasks (Lundh \& Czyzykow-Czarnocka, 2001), participants completed questionnaires 
after the task. Then, a moderation analysis was conducted. Interactive effects were investigated using trait and state scales, like Egloff and Hock (2001).

\section{EXPERIMENT 1}

\section{Methods}

\section{Participants}

Participants were 65 undergraduate students who provided written informed consent. All had normal or corrected-to-normal vision. They were divided into two groups: short-duration $(n=41 ; 10$ men and 31 women; aged 18-22 years; mean age $=19.5$ years $)$ and long-duration $(n=24 ; 9$ men and 15 women; aged $18-22$ years; mean age $=19.6$ years).

\section{Measures}

After the experiments, participants completed the State-Trait Anxiety Inventory-Trait (STAI-T) and State Forms (STAI-S). STAI-T and STAI-S are widely used general questionnaires to measure the degree of anxiety (Shimizu \& Imae, 1981; Spielberger et al., 1970). They comprise 20 items measured on a 4-point scale. The scales each have high internal consistency (STAI-T: $\alpha=.85$; STAI-S: $\alpha=.87$ ) and moderate test-retest reliability (STAI-T: $r=.80$; STAI-S: $r=.69$; Shimizu \& Imae, 1981).

\section{Stimuli and Procedure}

An orienting change-detection task was used to measure VSWMC (Vogel et al., 2005). Participants were briefly shown an array of visual stimuli to encode and maintain it for a later task (Fig. 1). The memory arrays consisted of four, eight, or twelve red bars $\left(0.12^{\circ} \times 0.52^{\circ}\right)$, which were selected randomly from a set of four orientations (vertical, horizontal, left $45^{\circ}$, and right $45^{\circ}$ ). All stimuli were presented within a $9.8^{\circ} \times$ $7.3^{\circ}$ region on a monitor with a grey background; the items were separated by at least $2.0^{\circ}$ (center to center). Stimulus positions were randomized for each trial.

On each trial, participants were required to see a central fixation cross for 400 to $600 \mathrm{~ms}$; then, a memory array was presented for $100 \mathrm{~ms}$ in the short-duration group and 500 or $1000 \mathrm{~ms}$ in the longduration group. The durations in the long-duration group were randomly selected and each duration was selected equally often. After a $900 \mathrm{~ms}$ blank interval, a test array was presented until participants responded. The test array was either identical to the original memory array or differed by one orientation, which was chosen from four orientations (vertical, horizontal, left $45^{\circ}$, and right $45^{\circ}$ ). Participants were required to press one of two buttons to indicate whether the two arrays were identical or different. In $50 \%$ of the trials, the test array was identical to the memory array. In the short-duration group, there were 80 trials in each set size $(N=240$ trials). In the long-duration group, there were 60 trials in each set size in each duration condition $(N=360$ trials $)$.

To investigate individual differences in memory capacity, participants' VSWMC was calculated by Kvalues following a standard formula (Cowan, 2001) $-\mathrm{K}=\mathrm{S}(\mathrm{H}-\mathrm{F})$ - where $\mathrm{K}$ is the memory capacity, $\mathrm{S}$ is the size of the array, $\mathrm{H}$ is the observed hit rate, and $\mathrm{F}$ is the false alarm rate. The maximum $\mathrm{K}$-value is the size of the array.

\section{Results}

The mean K-values at set-sizes 4, 8, and 12 are presented in Table 1. At set-size 4, $\mathrm{K}$-values of some participants reached the maximum (i.e., 4 items). In accordance with previous studies, to capture individual differences, I focused on the average K-values for 
(a)

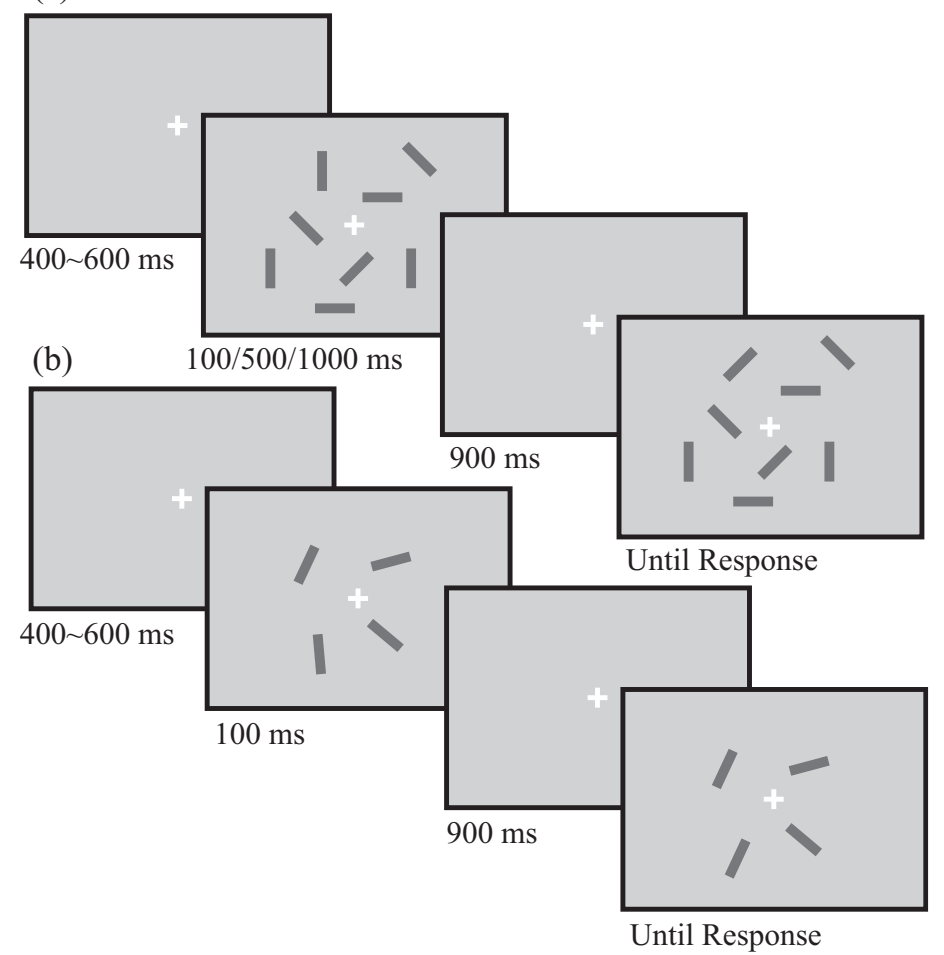

Fig. 1. Examples of the Change-Detection Tasks in (a) Experiment 1 and (b) Experiment 2

Table 1. Average K-Values for Visual Spatial Working Memory Capacity in Experiment 1 (Standard Errors in Parentheses)

\begin{tabular}{lcccc}
\hline & Set Size 4 & Set Size 8 & Set Size 12 & Average K-value \\
\hline \multirow{2}{*}{ Short Duration } & 3.32 & 3.46 & 3.37 & 3.41 \\
\multirow{2}{*}{ Long Duration } & $(0.41)$ & $(1.10)$ & $(1.42)$ & $(1.08)$ \\
$500 \mathrm{~ms}$ & & & & \\
& 3.42 & 4.27 & 3.78 & 4.03 \\
$1000 \mathrm{~ms}$ & $(0.47)$ & $(1.22)$ & $(1.75)$ & $(1.30)$ \\
& 3.58 & 4.57 & 3.87 & 4.22 \\
& $(0.32)$ & $(1.18)$ & $(2.10)$ & $(1.52)$ \\
\hline
\end{tabular}

Note. Average K-values were averaged with set-size 8 and 12 .

set-sizes 8 and 12 (Machizawa \& Driver, 2011; Moriya \& Sugiura, 2012). In addition, the average K-values for 500 and 1000-ms durations in the long-duration group did not differ, $t(23)=0.78, p=.44, d=.14$. Therefore, in the long-duration group, K-values for 500 and 1000-ms durations were averaged. I investigated the gender difference of 
Table 2. Correlations Between Trait Anxiety, State Anxiety, and Average K-Values in Experiment 1 (Lower and Upper of 95\% Confidence Interval in Parentheses)

\begin{tabular}{|c|c|c|c|c|}
\hline & \multicolumn{2}{|c|}{ Short-Duration Group $(n=41)$} & \multicolumn{2}{|c|}{ Long-Duration Group $(n=24)$} \\
\hline & State Anxiety & Average K-value & State Anxiety & Average K-value \\
\hline Trait Anxiety & $\begin{array}{c}.368 * \\
(.068, .607)\end{array}$ & $\begin{array}{c}.259 \\
(-.053, .525)\end{array}$ & $\begin{array}{c}.390 \\
(-.016, .685)\end{array}$ & $\begin{array}{c}.064 \\
(-.348, .456)\end{array}$ \\
\hline State Anxiety & - & $\begin{array}{c}.066 \\
(-.247, .366)\end{array}$ & - & $\begin{array}{c}-.067 \\
(-.458, .346)\end{array}$ \\
\hline
\end{tabular}

$* p<.05$.

Table 3. Hierarchical Linear Regression Analysis Predicting Average K-values Under Each Group in Experiment 1

\begin{tabular}{|c|c|c|c|c|c|c|c|c|c|c|}
\hline & \multicolumn{5}{|c|}{ Short-Duration Group } & \multicolumn{5}{|c|}{ Long-Duration Group } \\
\hline & $B$ & $S E B$ & $\beta$ & $\Delta F$ & $\Delta R^{2}$ & $B$ & $S E B$ & $\beta$ & $\Delta F$ & $\Delta R^{2}$ \\
\hline Step 1 & & & & 1.39 & .068 & & & & 0.15 & .014 \\
\hline Trait Anxiety & .030 & .018 & .271 & & & .012 & .027 & .106 & & \\
\hline State Anxiety & -.005 & .023 & -.034 & & & -.016 & .036 & -.108 & & \\
\hline Step 2 & & & & 9.83 & $.196^{*}$ & & & & 13.23 & $.393 *$ \\
\hline Trait Anxiety & -.004 & .020 & -.036 & & & -.009 & .022 & -.083 & & \\
\hline State Anxiety & .029 & .023 & .214 & & & -.003 & .028 & -.019 & & \\
\hline Trait $\times$ State Anxiety & -.007 & .002 & $-.544 *$ & & & -.009 & .003 & $-.651^{*}$ & & \\
\hline
\end{tabular}

$* p<.05$.

average K-values in short- and long-duration groups, because women have been reported to show high-anxiety scores (McLean \& Anderson, 2009) and gender a gender difference is an important factor in anxiety. There were no significant differences in short-duration group, $t(39)=0.190, p=.851, d=.068$, Male Average $=3.36, S D=0.74$, Female Average $=3.43, S D=1.18$, and long-duration group, $t(22)=0.896, p=.380, d=.365$, Male Average $=3.82, S D=1.44$, Female Average $=4.30, S D=1.18$. Therefore, in the following analysis, I did not include gender differences. Correlations between trait anxiety, state anxiety, and K-values in the both short- and long-duration groups are shown in Table 2.

To investigate the interactive effects of trait and state anxiety on VSWMC, a hierarchical regression analysis was conducted to predict averaged K-values using traitand state-anxiety scores. First, all independent variables were centered on the grand mean to minimize problems associated with multicollinearity and to scale the variables meaningfully (Aiken \& West, 1991; Bauer \& Curran, 2005). In Step 1 (main effects), 
(a)

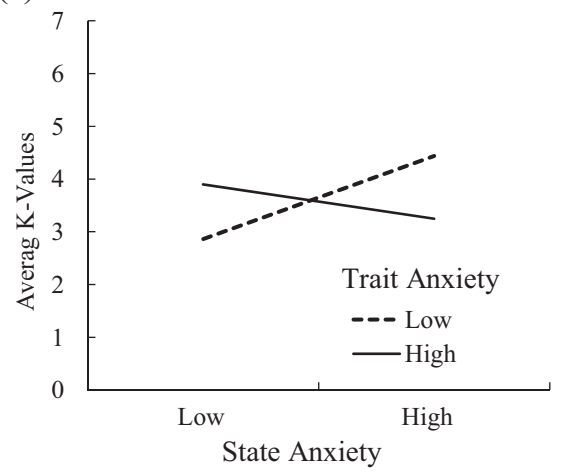

(b)

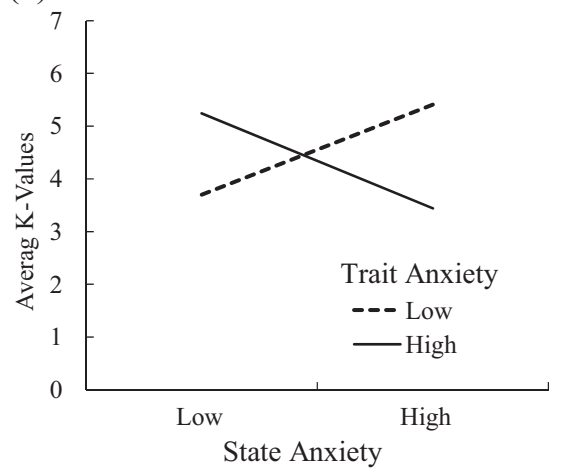

Fig. 2. The Interactive Effects of Trait- and State-Anxiety on Average K-Values in (a) the Short-Duration and (b) the Long-Duration Groups in Experiment 1

Note. All predicted scoress are calculated at $\pm 1 S D$ from the mean scores.

trait- and state-anxiety scores were entered; then, in Step 2 (interaction effect), the trait anxiety by state anxiety interaction was entered. Table 3 shows that the final model was significant in both the short-, $F(3,37)=4.415, p=.009, R^{2}=.264$, Adj. $R^{2}=.204$, and long-duration groups, $F(3,20)=4.567, p=.014, R^{2}=.407$, Adj. $R^{2}=.318$. The interaction of trait- and state-anxiety was significant. Fig. 2 shows the interaction by the simple slopes at one standard deviation $(S D)$ above and below the mean trait anxiety. The results in the short-duration group indicated that the simple slope for low trait anxiety was significant, $B=.098, \beta=.727, p=.015$, but that for high trait anxiety was not significant, $B=-.041, \beta=-.300, p=.093$. Further, the simple slope for low state anxiety was significant, $B=.052, \beta=.477, p=.006$, but that for high state anxiety was not significant, $B=-.060, \beta=-.550, p=.077$. In the long-duration group, the results were similar to the short-duration group and more clear than that. The simple slopes for low and high trait anxiety were both significant, $B=.101, \beta=.668, p=.029 ; B=-.107$, $\beta=-.706, p=.010$, respectively, and the simple slopes for low and high state anxiety were also both significant, $B=.068, \beta=.604, p=.017 ; B=-.087, \beta=-.770, p=.020$, respectively.

Even when combining participants in both short- and long-duration groups, the results were consistent. Trait anxiety was significantly correlated with state anxiety, $r=$ $.368, p=.003$, while K-values were not correlated with trait anxiety, $r=.201, p=.11$, or state anxiety, $r=-.001, p=.99$. The hierarchical regression analysis predicting averaged $\mathrm{K}$-values using the trait- and state-anxiety scores showed that the final model with interaction was significant, $F(3,61)=7.642, p=.001, R^{2}=.273$, Adj. $R^{2}=.237$. The interaction between trait- and state-anxiety was significant, $B=-.007, \beta=-.531, p=$ .001 . The simple slopes for low and high trait anxiety were both significant, $B=.091, \beta$ $=.616, p=.003 ; B=-.065, \beta=-.442, p=.003$, respectively, and the simple slopes for low and high state anxiety were also both significant, $B=.060, \beta=.524, p=.001 ; B=$ $-.061, \beta=-.533, p=.014$, respectively. 


\section{DisCUSSION}

In Experiment 1, the interactive effects of trait and state anxiety on VSWMC were investigated. During high state anxiety, VSWMC decreased with trait anxiety. In high trait-anxious individuals, VSWMC decreased with state anxiety, whereas it increased with state anxiety in low anxious individuals. Unexpectedly, during low state anxiety, VSWMC increased with trait anxiety. These results were clearly observed in the longduration group.

These results were consistent with the hypothesis. Previous studies showed that executive function, or top-down control, was impaired during both high trait and high state anxiety (Egloff \& Hock, 2001; Hur et al., 2015; Rutherford et al., 2004). On the other hand, low-trait-anxious individuals might show increased executive function in high state-anxiety situations (Ursache \& Raver, 2014). These results suggested that, during high state anxiety, top-down control is impoverished in high trait-anxious individuals but enhanced in low trait-anxious individuals. Consistent with this suggestion, the results of the present study showed low VSWMC during high state anxiety in individuals with high trait anxiety but high VSWMC during the same condition in individuals with low trait anxiety.

Increased VSWMC with trait anxiety at low state anxiety was not hypothesized. This result suggests that, during low state anxiety, high trait-anxious individuals utilize top-down processing more compared with low anxious individuals. Several previous neuroimaging studies support this finding (Bijsterbosch et al., 2015; Crocker et al., 2012). Crocker et al. (2012) showed that increased trait negative affect was associated with "increased" activation of the DLPFC during "low" state anxiety. Resting-state f-MRI studies also showed the increased functional connectivity between the ventromedial PFC and the amygdala in trait anxious individuals under safe conditions (Bijsterbosch et al., 2015). These results suggest that, during low state anxiety, high trait-anxious individuals excessively use top-down processing even if it is not necessary. The present results are consistent with this idea that during low state anxiety, high trait-anxious individuals excessively controlled top-down processing, which led to high VSWMC. The results required replication in Experiment 2.

The results were clearly observed in the long-duration group. Some previous studies have shown that VSWMC was independent of encoding duration (Vogel et al., 2001), whereas other studies indicated that VSWMC increased with encoding duration (Eng et al., 2005). Long encoding duration may be necessary for adequate encoding when participants need to hold a lot of stimuli and more complex stimuli in mind. In the present study, the oriented bars were not complex stimuli. However, because stimuli were presented more than limitation of the VSWMC, sufficient encoding duration might be necessary. In the short-duration group, VSWMC decreased because of insufficient encoding duration and differences between trait- and state-anxiety might be also decreased.

The role of top-down control in the change-detection task concerns not only attentional selection but also efficient allocation of VSWMC resources (Fougnie et al., 2016). Previous studies have shown that VSWMC can be flexibly allocated to stimuli 
depending on goals (Bays \& Husain, 2008; Zhang \& Luck, 2008). When individuals need to retain precise information (i.e., high-quality visual spatial working memory [VSWM]), they can allocate more VSWM resources to the stimuli at the cost of VSWM quantity. The allocation of VSWM resources is manipulated under top-down control (Fougnie et al., 2016).

In Experiment 1, participants might not have needed to retain precise information. In that experiment, target stimuli were rotated 45 or 90 degrees from their counterpart, a circumstance in which VSWM precision would not be important and coarse precision would be enough for stimuli detection change (Machizawa \& Driver, 2011; Machizawa et al., 2012; Murray et al., 2012). That is, the task in Experiment 1 was a "low-precision condition." In a high-precision task, more effort for controlling allocation of VSWM resources would be necessary, and the effects of top-down control would be more conspicuous. Consequently, in Experiment 2, I examined the interactive effects of trait and state anxiety on VSWMC by increasing precision.

\section{EXPERIMENT 2}

In Experiment 2, participants were required to retain precise information in the same paradigm as Experiment 1. There were two precision conditions: medium and high. In the medium-precision condition, the target stimulus was rotated 30 degrees from its counterpart; whereas, in the high-precision condition, it was rotated 15 degrees. In these conditions, participants needed to control allocation of visual working memory (VWM) resources more effortfully. Therefore, top-down control would influence VSMWC. The hypothesis was that, during high state anxiety, VSWMC would decrease with trait anxiety; however, during low state anxiety, VSWMC would increase with trait anxiety. Further, in high trait-anxious individuals, VSWMC would decrease with state anxiety, whereas it would increase with state anxiety in low trait-anxious individuals.

\section{MethodS}

\section{Participants}

Participants were 31 undergraduate students ( 9 men and 22 women; aged 18-22 years, mean $=19.6$ years) who provided informed consent. All had normal or corrected-to-normal vision.

\section{Measures}

After the experiments, participants completed the STAI-T and STAI-S (Shimizu \& Imae, 1981; Spielberger et al., 1970).

\section{Stimuli and Procedure}

The orienting change-detection task was identical to those in Experiment 1, with the following modifications (Fig. 1). In the memory array, participants were presented with a brief array of 2, 4, or 6 red bars $\left(0.12^{\circ} \times 0.52^{\circ}\right)$ selected randomly from 36 orientations $\left(5^{\circ}\right.$ possible steps $)$ for $100 \mathrm{~ms}$. Participants were instructed to remember the items. Memory for these red items was tested $900 \mathrm{~ms}$ later with a test array that 
was either identical to the original memory array or differed by one orientation. There were two conditions: high and medium precision. In the high-precision condition, the differed item was rotated $15^{\circ}$ clockwise or non-clockwise from its counterpart; whereas, in the medium-precision condition, it was rotated $30^{\circ}$ clockwise or non-clockwise. The rotated orientation and degrees of the different item were randomly selected, and each stimulus was presented equally often. There were 60 trials in each set size and precision condition $(N=360$ trials). Individuals' memory capacity was again calculated by $\mathrm{K}$-values following a standard formula (Cowan, 2001).

\section{RESULTS}

The mean K-values at set-sizes 2, 4, and 6 are presented in Table 4. In the highprecision condition, the average $\mathrm{K}$-values for set-sizes 2,4 , and 6 were used. On the other hand, in the medium-precision condition, the average K-values for set-sizes 4 and 6 were utilized because at set-size 2, the K-values of some participants nearly reached the maximum (i.e., 2 items). Because there were no significant gender differences in highprecision condition, $t(30)=0.586, p=.562, d=.218$, male average $=0.72, S D=0.37$, female average $=0.80, S D=0.39$, and medium-precision condition, $t(30)=0.372, p=$ $.712, d=.138$, male average $=2.05, S D=0.97$, female average $=1.94, S D=0.63$, I did not include gender differences in the following analysis. The correlations between trait anxiety, state anxiety, and K-values in high- and medium-precision conditions are shown

Table 4. Average K-Values for Visual Spatial Working Memory Capacity in Experiment 2 (Standard Errors in Parentheses)

\begin{tabular}{lcccc}
\hline & Set Size 2 & Set Size 4 & Set Size 6 & Average K-value \\
\hline High Precision & 0.84 & 0.76 & 0.70 & 0.77 \\
& $(0.29)$ & $(0.53)$ & $(0.69)$ & $(0.39)$ \\
Medium Precision & 1.49 & 2.00 & 1.93 & 1.97 \\
& $(0.38)$ & $(0.71)$ & $(0.94)$ & $(0.75)$ \\
\hline
\end{tabular}

Note. Average K-values were averaged with set-size 2, 4, and 6 in the high-precision condition, and with 4 and 6 in the medium-precision condition.

Table 5. Correlations Between Trait Anxiety, State Anxiety, and Average K-values in Experiment 2 (Lower and Upper of 95\% Confidence Interval in Parentheses)

\begin{tabular}{lccc}
\hline State Anxiety & $\begin{array}{c}\text { Average K-value in } \\
\text { High Precision }\end{array}$ & $\begin{array}{c}\text { Average K-value in } \\
\text { Medium Precision }\end{array}$ \\
\hline Trait Anxiety & $(-.034, .600)$ & .176 & .189 \\
State Anxiety & - & $(-.184, .494)$ & $(-.171, .505)$ \\
& $-.394 *$ & -.134 \\
& & $(-.653,-.052)$ & $(-.461, .225)$ \\
\hline
\end{tabular}

$* p<.05$. 
in Table 5 .

For the interactive effects of trait and state anxiety on VSWMC, a hierarchical regression analysis was conducted to predict averaged K-values using trait- and stateanxiety scores (the same as in Experiment 1). Table 6 shows that the final model was significant in the high- and medium-precision conditions: $F(3,27)=3.580, p=.027, R^{2}$ $=.285$, Adj. $R^{2}=.205 ; F(3,27)=3.169, p=.040, R^{2}=.260$, Adj. $R^{2}=.178$, respectively. However, the interaction between trait and state anxiety was only significant in the medium-precision condition. Fig. 3 shows the interaction by the simple slopes at one $S D$ above and below the mean trait anxiety. The simple slope analysis was conducted only for the medium-precision condition. The results indicated that the simple slope for high

Table 6. Hierarchical Linear Regression Analysis Predicting Average K-values Under Each Condition in Experiment 2

\begin{tabular}{|c|c|c|c|c|c|c|c|c|c|c|}
\hline & \multicolumn{5}{|c|}{ High-Precision Condition } & \multicolumn{5}{|c|}{ Medium-Precision Condition } \\
\hline & $B$ & $S E B$ & $\beta$ & $\Delta F$ & $\Delta R^{2}$ & $B$ & $S E B$ & $\beta$ & $\Delta F$ & $\Delta R^{2}$ \\
\hline Step 1 & & & & 4.91 & $.259^{*}$ & & & & 1.26 & .082 \\
\hline Trait Anxiety & .013 & .006 & $.354 *$ & & & .019 & .013 & .269 & & \\
\hline State Anxiety & -.021 & .007 & $-.482 *$ & & & -.017 & .016 & -.204 & & \\
\hline Step 2 & & & & 0.95 & .025 & & & & 6.50 & $.178^{*}$ \\
\hline Trait Anxiety & .012 & .006 & .323 & & & .013 & .013 & .187 & & \\
\hline State Anxiety & -.018 & .008 & $-.413 *$ & & & -.002 & .016 & -.021 & & \\
\hline Trait $\times$ State Anxiety & -.001 & .001 & -.172 & & & -.004 & .002 & $-.458^{*}$ & & \\
\hline
\end{tabular}

$* p<.05$.

(a)

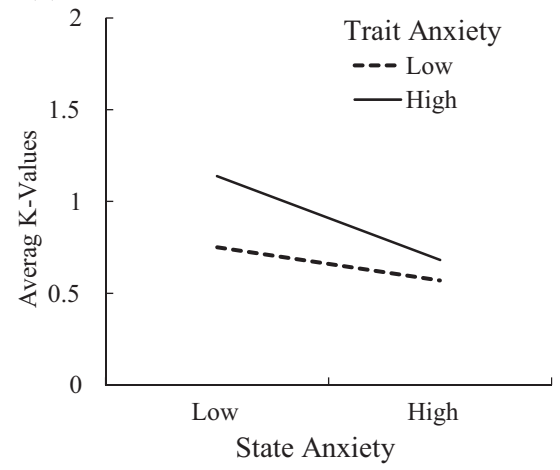

(b)

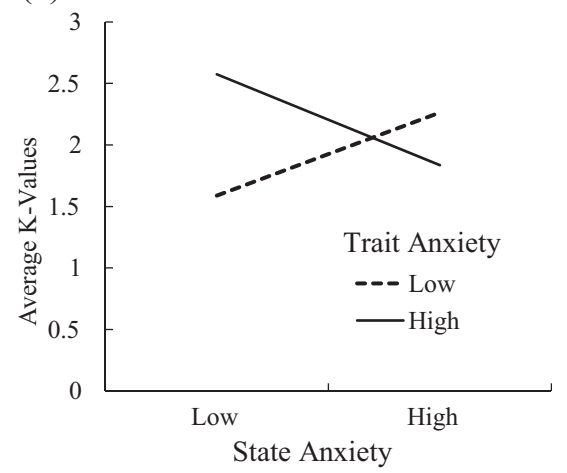

Fig. 3. The Interactive Effects of Trait- and State-Anxiety on Average K-Values in (a) the High-Precision and (b) the Medium-Precision Conditions in Experiment 2

Note. All predicted scoress are calculated at $\pm 1 S D$ from the mean scores. 
trait anxiety was significant, $B=-.042, \beta=-.496, p=.024$, but that for low trait anxiety was not, $B=.038, \beta=.453, p=.156$. The simple slope for low state anxiety was also significant, $B=.047, \beta=.661, p=.008$, but that for high state anxiety was not, $B=$ $-.020, \beta=-.288, p=.310$.

\section{Discussion}

In Experiment 2, the interactive effects of trait and state anxiety on VSWMC under medium- and high-precision conditions were investigated. In the medium-precision condition (i.e., 30-degree rotation), VSWMC decreased with state anxiety among high trait-anxiety individuals. During low state anxiety, VSWMC increased with trait anxiety. Further, in the high-precision condition (i.e., 15-degree rotation), VSWMC decreased with state anxiety, while it increased with trait anxiety.

The results of the medium-precision condition were consistent with Experiment 1- high trait-anxious individuals showed low VSWMC during high state anxiety and high VSWMC during low state anxiety. During both high trait and state anxiety, individuals have difficulty utilizing top-down control (Egloff \& Hock, 2001; Hur et al., 2015; Rutherford et al., 2004), which may lead to low VSWMC. However, the interactive effects of state anxiety on low trait anxiety decreased. The increased VSMWC in the low trait-anxious individuals at high state anxiety was not significant. Moreover, in the high-precision condition, even in low trait-anxious individuals, VSWMC decreased with state anxiety. That is, the more precise the retained information is, the lower the level of the interactive effects of state anxiety.

State anxiety may not work efficiently for the processing of precise information. To retain precise information, VSWM-resource allocation should be focused on a few stimuli (Fougnie et al., 2016), whereas increased state anxiety would enhance wide allocation of VSWM resources. Under threatening situations, enhanced bottom-up processing and widely allocated resources would be helpful to efficiently detect potential threatening stimuli (Eysenck et al., 2007). As Pacheco-Unguetti et al. (2010) showed, state anxiety increases alerting function related to enhanced bottom-up processing and high vigilance, which may lead to wide allocation of resources. However, this would be inefficient to retain precise information. Therefore, in the high-precision condition, high state anxiety would not increase VSWMC.

\section{General Discussion}

The present study investigated the interactive effects of trait and state anxiety on VSWMC using a change-detection task. The precision of VSWM representations was manipulated by changing the rotation of the target stimulus. In the low-precision condition in Experiment 1, the orientation of stimuli was always vertical, horizontal, left $45^{\circ}$, or right $45^{\circ}$, and the target stimulus was rotated 45 or 90 degrees from its 
counterpart; then, in Experiment 2, the orientation of stimuli was selected from 36 orientations ( $5^{\circ}$ possible steps), and the target stimulus was rotated, from its counterpart, 30 degrees in the medium-precision condition and 15 degrees in the high-precision condition. In the low-precision condition, VSWMC decreased with trait anxiety during high state anxiety, whereas it increased with trait anxiety during low state anxiety. In high trait-anxious individuals, VSWMC decreased with state anxiety, whereas it increased with state anxiety in low anxious individuals. In the medium-precision condition, VSWMC decreased with state anxiety in high trait-anxious individuals and it increased with trait anxiety during low state anxiety, whereas the effects of state anxiety were decreased in low trait-anxious individuals. Moreover, in the high-precision condition, the interactive effects of trait and state anxiety were eliminated: VSWMC decreased with state anxiety and increased with trait anxiety.

Previous models suggested that trait anxiety is associated with impaired top-down control, whereas state anxiety is associated with enhanced bottom-up control (Bishop, 2007; Eysenck et al., 2007). Several previous studies have supported these models (Bishop, 2009; Bishop et al., 2007; Crocker et al., 2012; Pacheco-Unguetti et al., 2010), and others focused on the interactive effects of trait and state anxiety on cognitive functions. The trait-related impaired top-down control is observed during high state anxiety (Crocker et al., 2012; Egloff \& Hock, 2001; Hur et al., 2015; Rutherford et al., 2004), whereas top-down control is enhanced during high state anxiety in low traitanxious individuals (Bijsterbosch et al., 2015; Ursache \& Raver, 2014). The present results in the low-precision condition are consistent with these prior results. Because topdown control is necessary to efficiently retain a restricted number of stimuli in a changedetection task (Cusack et al., 2009; Linke et al., 2011), impaired top-down control in high trait-anxious individuals during high state anxiety leads to low VSWMC, whereas the enhanced top-down control in low trait-anxious individuals during high state anxiety leads to high VSWMC.

However, after increasing the information precision in VWM, the interactive effects of state anxiety on trait anxiety were diminished. State anxiety might play two distinct roles: enhanced top-down control and enhanced bottom-up processing. During high state anxiety, enhanced top-down processing works in low trait-anxious individuals, as seen in the present results, whereas enhanced bottom-up processing is also observed (PachecoUnguetti et al., 2010). The enhanced top-down control would enable individuals to efficiently process stimuli based on the goal, whereas the enhanced bottom-up processing would enable individuals to detect potential threatening stimuli. In the low-precision condition, both could produce interactive effects to increase VSWMC because enhanced top-down control enables individuals to retain a restricted number of stimuli (Cusack et al., 2009; Linke et al., 2011) and enhanced bottom-up processing could widen allocation of VSWM resources. However, the wide allocation of VSWM resources might compete with top-down control in the high-precision condition because focused VSWM-resource allocation to a few stimuli would be necessary to retain precise information (Fougnie et al., 2016). Therefore, high precision information could not be retained during high state anxiety. 
The present study had several limitations. First, the sample size was insufficient, especially in Experiment 2. In Experiment 1, I confirmed that the present results were observed when combining the data in the short- and long-duration groups. Although this analysis increased the sample size, further studies should include a larger number of participants under the same conditions. Second, state anxiety was not manipulated by a mood-induction procedure, but rather only measured by questionnaires. The previous study also assessed both state and trait anxiety with questionnaires (Egloff \& Hock, 2001). However, this procedure is problematic in that trait and state anxiety measured by questionnaires are generally highly correlated. A mood-induction procedure is more effective for investigating the effects of state anxiety. Third, the order of scales and experiments was not controlled. In the present study, participants completed the scales after the experiment, because answering questionnaires before a cognitive experiment might affect the performance of the task (Lundh \& Czyzykow-Czarnocka, 2001). The state anxiety assessed after the experiment might be strongly influenced from the task performance, whereas anxiety assessed before the experiment might reflect a taskunrelated mood. The former phenomenon, anxiety elicited by the task, is related to integral emotion, whereas the latter phenomenon, anxiety elicited by a task-unrelated mood, is related to incidental emotion, and these differences seem to affect several cognitive controls (Blanchette \& Richards, 2010; Inzlicht et al., 2015). Because in the present study, state-anxiety score might reflect integral anxiety, the correlation between trait and state anxiety might be low as compared with typical states. However, the present study could not clarify the different effects of integral and incidental anxiety on VSWMC.

In summary, the present results outline the interactive effects of trait and state anxiety on VSWMC. Trait anxiety does not necessarily reduce the VSWMC but increases the VSWMC depending on state anxiety. The distinct effects of trait anxiety on the VSWMC may help to explain inconsistent results of associations between anxiety and VSWMC under no-distractor conditions in the previous studies.

\section{DECLARATION OF INTEREST}

I declare that there are no conflicts of interest.

\section{REFERENCES}

Aiken, L. S., \& West, S. G. (1991). Multiple regression: Testing and interpreting interactions. Sage.

Amir, N., \& Bomyea, J. (2011). Working memory capacity in generalized social phobia. Journal of Abnormal Psychology, 120(2), 504-509. https://doi.org/10.1037/a0022849

Bauer, D., \& Curran, P. (2005). Probing interactions in fixed and multilevel regression: Inferential and graphical techniques. Multivariate Behavioral Research, 40(3), 373-400. https://doi.org/10.1207/ s15327906mbr4003_5

Bays, P. M., \& Husain, M. (2008). Dynamic shifts of limited working memory resources in human vision. 
Science, 321(5890), 851-854. https://doi.org/10.1126/science.1158023

Berggren, N., Curtis, H. M., \& Derakshan, N. (2017). Interactions of emotion and anxiety on visual working memory performance. Psychonomic Bulletin and Review, 24(4), 1274-1281. https://doi.org/10.3758/ s13423-016-1213-4

Bijsterbosch, J., Smith, S., \& Bishop, S. J. (2015). Functional connectivity under anticipation of shock: Correlates of trait anxious affect versus induced anxiety. Journal of Cognitive Neuroscience, 27(9), 1840-1853. https://doi.org/10.1162/jocn_a_00825

Bishop, S. J. (2007). Neurocognitive mechanisms of anxiety: An integrative account. Trends in Cognitive Sciences, 11(7), 307-316. https://doi.org/10.1016/j.tics.2007.05.008

Bishop, S. J. (2009). Trait anxiety and impoverished prefrontal control of attention. Nature Neuroscience, 12(1), 92-98. https://doi.org/10.1038/nn.2242

Bishop, S. J., Jenkins, R., \& Lawrence, A. D. (2007). Neural processing of fearful faces: Effects of anxiety are gated by perceptual capacity limitations. Cerebral Cortex, 17(7), 1595-1603. https://doi.org/10. 1093/cercor/bhl070

Blanchette, I., \& Richards, A. (2010). The influence of affect on higher level cognition: A review of research on interpretation, judgement, decision making and reasoning. Cognition and Emotion, 24(4), 561-595. https://doi.org/10.1080/02699930903132496

Cowan, N. (2001). The magical number 4 in short-term memory: A reconsideration of mental storage capacity. Behavioral and Brain Sciences, 24(1), 87-114. https://doi.org/10.1017/S0140525X01003922

Crocker, L. D., Heller, W., Spielberg, J. M., Warren, S. L., Bredemeier, K., Sutton, B. P., Banich, M. T., \& Miller, G. A. (2012). Neural mechanisms of attentional control differentiate trait and state negative affect. Frontiers in Psychology, 3, Article 298. https://doi.org/10.3389/fpsyg.2012.00298

Cusack, R., Lehmann, M., Veldsman, M., \& Mitchell, D. J. (2009). Encoding strategy and not visual working memory capacity correlates with intelligence. Psychonomic Bulletin \& Review, 16(4), 641-647. https://doi.org/10.3758/PBR.16.4.641

Egloff, B., \& Hock, M. (2001). Interactive effects of state anxiety and trait anxiety on emotional Stroop interference. Personality and Individual Differences, 31(6), 875-882. https://doi.org/10.1016/S01918869(00)00188-4

Eng, H. Y., Chen, D., \& Jiang, Y. (2005). Visual working memory for simple and complex visual stimuli. Psychonomic Bulletin \& Review, 12(6), 1127-1133. https://doi.org/10.3758/BF03206454

Eysenck, M. W., \& Derakshan, N. (2011). New perspectives in attentional control theory. Personality and Individual Differences, 50(7), 955-960. https://doi.org/10.1016/j.paid.2010.08.019

Eysenck, M. W., Derakshan, N., Santos, R., \& Calvo, M. G. (2007). Anxiety and cognitive performance: Attentional control theory. Emotion, 7(2), 336-353. https://doi.org/10.1037/1528-3542.7.2.336

Fougnie, D., Cormiea, S. M., Kanabar, A., \& Alvarez, G. A. (2016). Strategic trade-offs between quantity and quality in working memory. Journal of Experimental Psychology: Human Perception and Performance, 42(8), 1231-1240. https://doi.org/10.1037/xhp0000211

Hayes, S., Hirsch, C., \& Mathews, A. (2008). Restriction of working memory capacity during worry. Journal of Abnormal Psychology, 117(3), 712-717. https://doi.org/10.1037/a0012908

Hur, J., Miller, G. A., McDavitt, J. R. B., Spielberg, J. M., Crocker, L. D., Infantolino, Z. P., Towers, D. N., Warren, S. L., \& Heller, W. (2015). Interactive effects of trait and state affect on top-down control of attention. Social Cognitive and Affective Neuroscience, 10(8), 1128-1136. https://doi.org/10.1093/ scan/nsu163

Inzlicht, M., Bartholow, B. D., \& Hirsh, J. B. (2015). Emotional foundations of cognitive control. Trends in Cognitive Sciences, 19(3), 126-132. https://doi.org/10.1016/j.tics.2015.01.004

Leigh, E., \& Hirsch, C. R. (2011). Worry in imagery and verbal form: Effect on residual working memory capacity. Behaviour Research and Therapy, 49(2), 99-105. https://doi.org/10.1016/j.brat.2010.11.005

Linke, A. C., Vicente-Grabovetsky, A., Mitchell, D. J., \& Cusack, R. (2011). Encoding strategy accounts for individual differences in change detection measures of VSTM. Neuropsychologia, 49(6), 1476-1486. https://doi.org/10.1016/J.NEUROPSYCHOLOGIA.2010.11.034

Luck, S. J., \& Vogel, E. K. (1997). The capacity of visual working memory for features and conjunctions. Nature, 390(6657), 279-281. https://doi.org/10.1038/36846

Luck, S. J., \& Vogel, E. K. (2013). Visual working memory capacity: From psychophysics and neurobiology to individual differences. Trends in Cognitive Sciences, 17(8), 391-400. https://doi.org/10.1016/j.tics. 
2013.06.006

Lundh, L. G., \& Czyzykow-Czarnocka, S. (2001). Priming of the emotional stroop effect by a schema questionnaire. An experimental study of test order. Cognitive Therapy and Research, 25(3), 281-289. https://doi.org/10.1023/A:1010784412175

Machizawa, M. G., \& Driver, J. (2011). Principal component analysis of behavioural individual differences suggests that particular aspects of visual working memory may relate to specific aspects of attention. Neuropsychologia, 49(6), 1518-1526. https://doi.org/10.1016/j.neuropsychologia.2010.11.032

Machizawa, M. G., Goh, C. C. W., \& Driver, J. (2012). Human visual short-term memory precision can be varied at will when the number of retained items is low. Psychological Science, 23(6), 554-559. https://doi.org/10.1177/0956797611431988

McLean, C. P., \& Anderson, E. R. (2009). Brave men and timid women? A review of the gender differences in fear and anxiety. Clinical Psychology Review, 29(6), 496-505. https://doi.org/10.1016/ j.cpr.2009.05.003

Moran, T. P. (2016). Anxiety and working memory capacity: A meta-analysis and narrative review. Psychological Bulletin, 142(8), 831-864. https://doi.org/10.1037/bul0000051

Moriya, J. (2018). Attentional networks and visuospatial working memory capacity in social anxiety. Cognition and Emotion, 32(1), 158-166. https://doi.org/10.1080/02699931.2016.1263601

Moriya, J., \& Sugiura, Y. (2012). High visual working memory capacity in trait social anxiety. PLOS ONE, 7(4), Article e34244. https://doi.org/10.1371/journal.pone.0034244

Murray, A. M., Nobre, A. C., Astle, D. E., \& Stokes, M. G. (2012). Lacking control over the trade-off between quality and quantity in visual short-term memory. PLOS ONE, 7(8), Article e41223. https://doi.org/10.1371/journal.pone.0041223

Pacheco-Unguetti, A. P., Acosta, A., Callejas, A., \& Lupiáñez, J. (2010). Attention and anxiety: Different attentional functioning under state and trait anxiety. Psychological Science, 21(2), 298-304. https: //doi.org/10.1177/0956797609359624

Qi, S., Chen, J., Hitchman, G., Zeng, Q., Ding, C., Li, H., \& Hu, W. (2014). Reduced presentations capacity in visual working memory in trait anxiety. Biological Psychology, 103, 92-99. https://doi.org/10. 1016/j.biopsycho.2014.08.010

Qi, S., Ding, C., \& Li, H. (2014). Neural correlates of inefficient filtering of emotionally neutral distractors from working memory in trait anxiety. Cognitive, Affective, \& Behavioral Neuroscience, 14(1), 253-265. https://doi.org/10.3758/s13415-013-0203-5

Rutherford, E. M., MacLeod, C., \& Campbell, L. W. (2004). Negative selectivity effects and emotional selectivity effects in anxiety: Differential attentional correlates of state and trait variables. Cognition and Emotion, 18(5), 711-720. https://doi.org/10.1080/02699930341000121

Shimizu, H., \& Imae, K. (1981). State-Trait Anxiety Inverntory no nihongoban (daigakusei yo) no sakusei [Development of the Japanese edition of the Spielberger State-Trait Anxiety Inventory (STAI) for student use]. Japanese Journal of Educational Psychology, 29(4), 348-353. https://doi.org/10.5926/ jjep1953.29.4_348

Spielberger, C. D., Gorsuch, R., \& Lushene, R. (1970). Manual for the State-Trait Anxiety Inventory. Consulting Psychologist Press.

Stout, D. M., \& Rokke, P. D. (2010). Components of working memory predict symptoms of distress. Cognition and Emotion, 24(8), 1293-1303. https://doi.org/10.1080/02699930903309334

Ursache, A., \& Raver, C. C. (2014). Trait and state anxiety: Relations to executive functioning in an at-risk sample. Cognition and Emotion, 28(5), 845-855. https://doi.org/10.1080/02699931.2013.855173

Vogel, E. K., McCollough, A. W., \& Machizawa, M. G. (2005). Neural measures reveal individual differences in controlling access to working memory. Nature, 438(7067), 500-503. https://doi.org/ 10.1038 /nature 04171

Vogel, E. K., Woodman, G. F., \& Luck, S. J. (2001). Storage of features, conjunctions, and objects in visual working memory. Journal of Experimental Psychology: Human Perception and Performance, 27(1), 92-114. https://doi.org/10.1037/0096-1523.27.1.92

Williams, J. M. G., Watts, F. N., MacLeod, C., \& Mathews, A. (1988). Cognitive psychology and emotional disorders. Wiley.

Yoon, K. L., LeMoult, J., Hamedani, A., \& McCabe, R. (2018). Working memory capacity and spontaneous emotion regulation in generalised anxiety disorder. Cognition and Emotion, 32(1), 215-221. https: 
//doi.org/10.1080/02699931.2017.1282854

Zhang, W., \& Luck, S. J. (2008). Discrete fixed-resolution representations in visual working memory. Nature, 453(7192), 233-235. https://doi.org/10.1038/nature06860

(Manuscript received 25 March, 2019; Revision accepted 12 June, 2019; Released online in J-STAGE as advance publication 28 October, 2020) 